\section{The benefits of exercise programmes for pre- school children with autism spectrum disorders}

Children with autism spectrum
disorder (ASD) see the world
differently and can find it hard
to interact with both their
environment and other people.
Sport has many benefits for
mental and physical health,
and therefore Kelong Cai,
of Yangzhou Universitty,
hypothesised that sport may
offer a novel therapy for
pre-school children with ASD.
He implemented a 12 -week
mini-basketball programme
for children with ASD and
found that this improved social
communication, executive
function, and reduced repetitive
actions. This suggests that
exercise programmes should be
considered alongside current
therapeutic approaches for
children with ASD.

utism spectrum disorder (ASD) -

utism spectrum disorder (ASD)
is a lifelong developmental

these improvements. There are differences in the brains of people with and without other people. It can also affect the way people interact with the world around them. According to the latest statistics from the US Centers for Disease Control and Prevention (CDC) the prevalence. of ASD has increased to 1 in 54 children. Autism is a spectrum condition meaning that whilst all people with ASD share some similarities, everyone is affected differently The terms ASD and autism are often used interchangeably

EXERCISE AND WHITE MATTER As well as keeping people fit and healthy, being part of a sports team or taking part in any physical activity can increase self-esteem, develop social skills, and improve mental health and wellbeing. Whist sport is something that can be senson overload and the need for social communication, with the correct support it is something that can make a huge

difference to a child's life.

Studies have previously shown that physical activity programmes can improve social communication outcomes in children with autism. This includes improvements in social awareness and cognition, and emotional regulation. It is unclear, how whether the type of sport makes difference. Researchers have noticed that team sports with an individual component such as baseball or basketball, are good ASD. Specificaly peoplewith ASD tend made up of a collection of densely packed neurons and is surrounded by grey matter. Scientists have previously shown that differences in white matter in the brains of children with ASD were associated with impaired social communication skills. Grey matter contains fewer nerve cells and includes regions of the brain involved in sensory perception, emotions, decisionmaking, and self-control.

Given these differences, Kelong Cai, of Yangzhou University, explored the relationship between exercise and white matter in relation to social communication. around mini-basketball.

Mini-basketball is a simplified version of adult basketball that is suitably adapted forchildren. The gols are lowered the ball is smaller, the game is played on a crowding around the ball. The minibasketball training programme (MBTP) conducted by two certified physical educators and a collective teaching mode was used to facilitate social interaction and communication among participants while parent's participation in and attendance at exercise sessions was strongly encouraged.

The MBTP was designed to includ various levels of difficulty so that eachers could administer the session at also motivate children to progress to

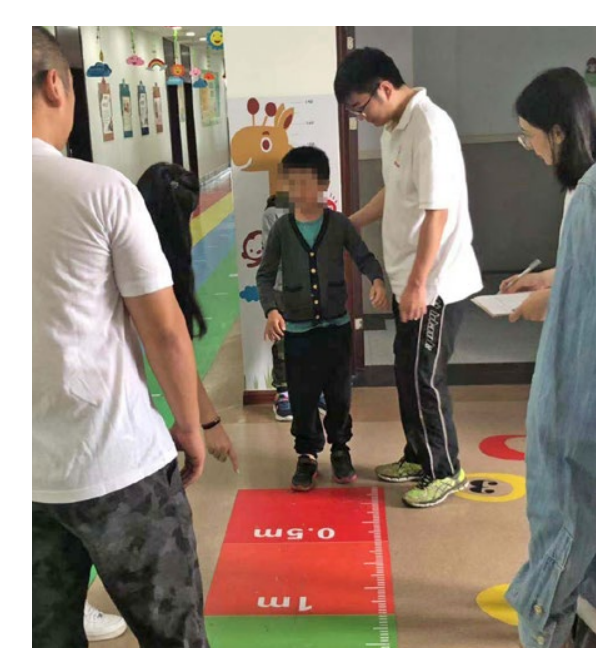

Children participated
training programme

higher levels in the course of the training programme. The 12-week MBTP was different targets.

THE BENEFITS OF SPORT FOR SOCIAL COMMUNICATION

The study recruited 29 children with ASD

who were aged three to six years old.

The children were split into two different

groups. One group participated in a $m$

consisting of five

for 12 weeks. The

control group were

with their normal

with their normal

The social the US Centers for Disease Control an Prevention $(C D C)$, the prevalence of ASD has increased to 1 in 54 children.

A new study investigated the communication skills of each childwe They also noticed changes in the white the progam the sills ind the end of the programme. These sklls involved things responding to other people appropriately, and understanding what people meant when they spoke Kelong Cai and

colleagues also used a specialist imaging device to take images of each child's brain at the beginning and the end of the study. group. Specifically, these changes seemed to be in areas of the brain that are known linked to social communication impairments in individuals with ASD.

HOW ELSE CAN SPORT HELP CHILDREN WITH AUTISM? As well as changes in communication and

mini-balfects of a 12-week mi-basketball training programme symptoms of pre-schol chile ASD. As with the previous study 59 pre-school children with ASD were plit into two groups: an exercise and a control group.

Different outcome measures were

PHASE

$$
\text { OAL }
$$

CONTENT

DURATION

PHASE I Standardise classroom routines Classroom routines (taking turns, waiting, obeying, etc.) 2 weeks Increase children's interest in mini-basketball Simple basketball training (roll and throw the ball, etc.)

PHASE II Improve children's mini-basketball skills Basic basketball skill (dribbling, passing, shooting, etc)

Improve social communication skills

Improve children's cooperative ability
social skills, and collectivisation (passing and catching ball, relay racing, etc) Group game based on mini-basketball (basketball-dribbling relay, basketball-passing relays,
basket-moving shooting, playing ducks, etc.)

8 weeks

Table 1. Mini-basketball training programme (MBTP) protocol. 12 -week MBTP was arranged for ASD children (40 min $\times 5$ sessions per week $\times 12$ weeks). Each
session included 4 stages of (a) 5 -min warm-up, (b) 20-min basic basketball skill learning, (c) 10-min basketball games, and (d) 5 -min cool-down (Table 2, overleaff) 


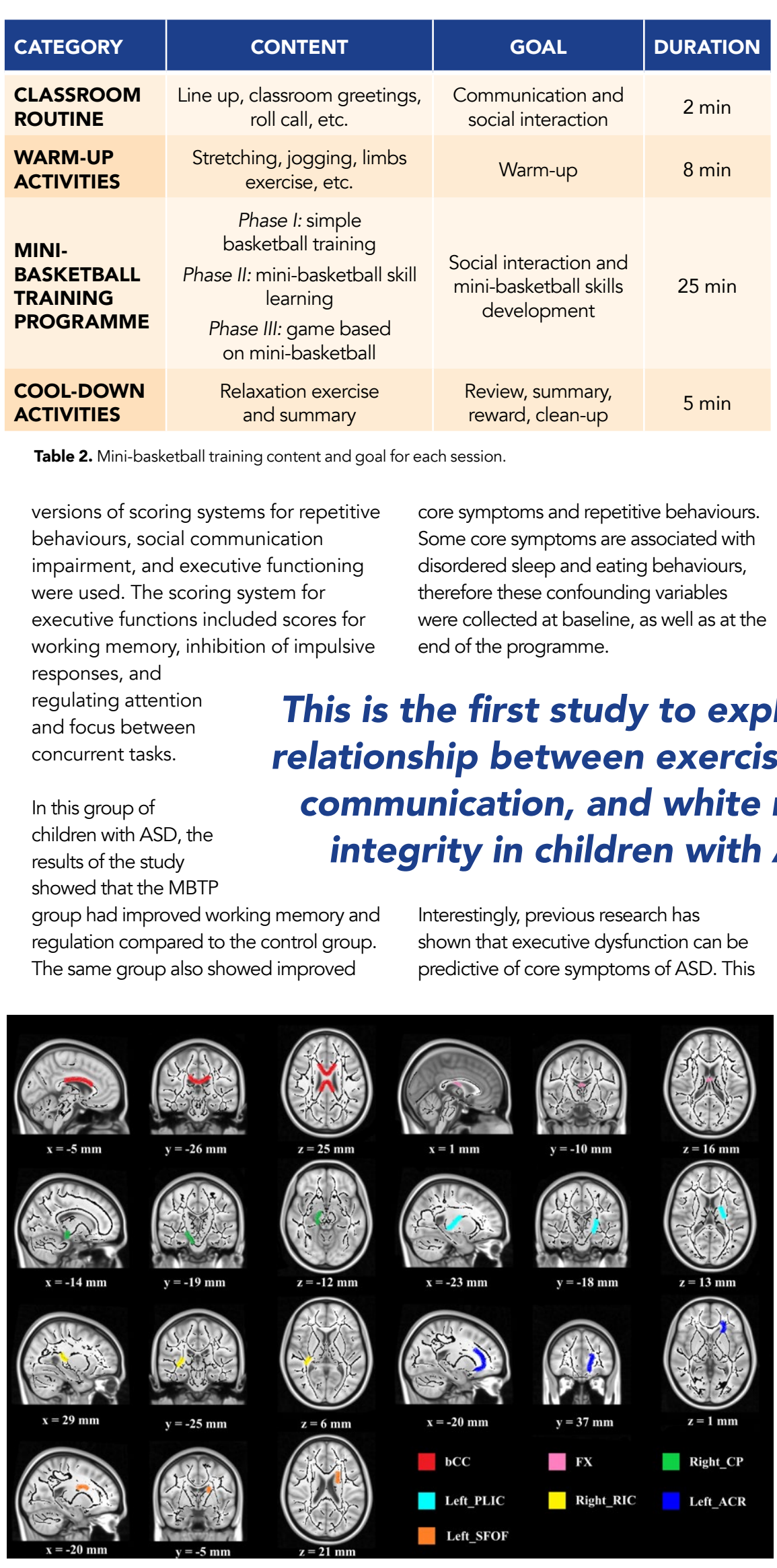

Demonstration of brain regions with white matter integrity increases after MBTP. Abbreviations

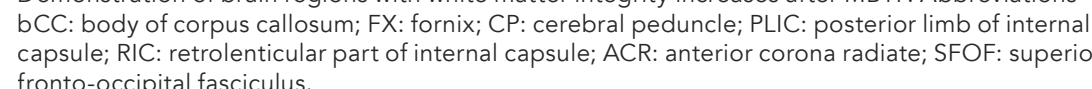

dds further support for Kelong Cai's work which focuses on pre-schoolers, as early . functioning in children with ASD.

Kelong Cai suggests that the benefits of exercise programmes might be linked to the cognitive demands placed on the brain during sports such as minibasketball, something known as the guidance effect'. For example, MBTP requires the children to learn new, complex motor skills. These new skills require the brain to work hard, and the demands placed on the brain may indirectly affect executive functions. However, further work is required to fully investigate this.

Alternatively, Cai explains that the release of neurochemicals, such as brainophic factor, could be fluencing cognitive functions.

re the ANEW APPROACH

NEW APPROACH SPECTRUM DISORDER This is the first study to explore the relationship between exercis

social communication, and white matter integrity in children with ASD. Although the sizes of the studies were relatively small, Kelong Cai emphasises that exercise can make a significant neurlological difference for children with autism and that this correlates with an Furthermore, most other studies have ASD, rather than pre-school children is well known that early life is a critical period for growth and development, therefore early intervention may be more effective than engaging with therapies later in life.

Currently, there is no pharmacological therapy for children with ASD. Instead, behavioural therapies are used, but these can be difficult for some families to access, and may only result in shorter term changes in ASD-related benviours. Exerlise programmes could offer an alternative therapy tha improves cognitive function and the

\section{Behind the Research}

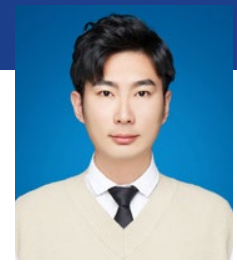

Kelong Cai

$\frac{\text { MX120170353@yzu.edu. }}{\mathrm{T}:+8615371334407}$

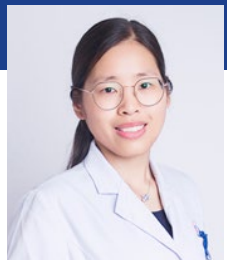

Zhimei Liu

E: DX120200076@yzu.edu.co

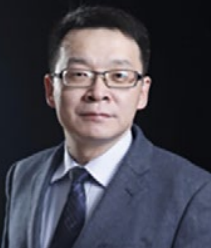

Wei Wang

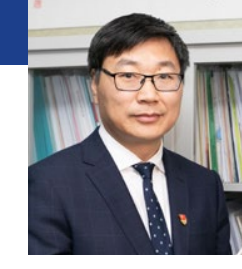

Aiguo Chen E: agchen@yzu.edu.cn
Research Objectives

Kelong Cai has studied the effects of exercise programmes

\section{Detail}

Addres

College of Physical Education Yangzhou University, Yangzhou,
No. 196, Huayang West Road Hanjiang District, Yangzhou, 225127 Bio

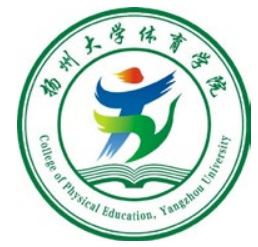
research of exercise o promote the rehabilitation of autistic children.

Zhimei Liu is a doctoral candidate at the College of Physica Education, Yangzhou University. She has been engaged rin clinical and scientific esearch work related to children's direction is sports rehabilitation and brain improvement of exceptional children.

Dr Wei Wang is a senior professor at Affiliated Hospital the en and functional bran field of neurologic impairment, especially in the function diagnosis of diseases. He has published a number of Parkinson's disease and cerebrovascular disease Dr Aiguo Chen is a professor at the College of Physical education from the Beiiing Normal University. He is the Changijiang scholar of China. His research focuses on exercise and brain in children and adolescents.

Funding

We would like to thank the National Natural Science Foundation of China (31771243) and the Fok Ying Tong (a)

We would like to thank Aiguo Chen and Zhimei Liu for their help with different aspects of this study and express our sincerest appreciation to the shildren and participate in this study. core member of the Institute of Sports, Exercise and Brain, aging. He has made outstanding contributions to the

\section{T: +8613952751176} Basketball Training Program on Executive Functions and Core

\section{References}

Cai, K Yu, Q Herold, F Liu, Z Wang, J et al (2020). MiniBasketball Training Program Improves Social Communication Sciences, 10(11), 803. https://doi.org/10.3390/ brainsci10110803

Cai, KL Wang, JG Liu, ZM Zhu, LN et al (2020). Mini-Basketball Training Program Improves Physical Fitness and Social Com doi.org/10.2478/hukin-2020-0007

Wang, JG Cai, KL Liu, ZM et al (2020). Effects of MiniSymptoms among Preschool Children with Autism Spectrum Disorders. Brain S
brainsci10050263

\section{Personal Response}

Did the changes persist after the exercise programme was stopped - ie, do you know how lon
the exercise programme might last?

The research we are currently conducting will evaluate Then with ASD 3 months after the exercise intervention is stopped. According to the preliminary data, the exercise couts yet published this work. In the future, we will conduct more

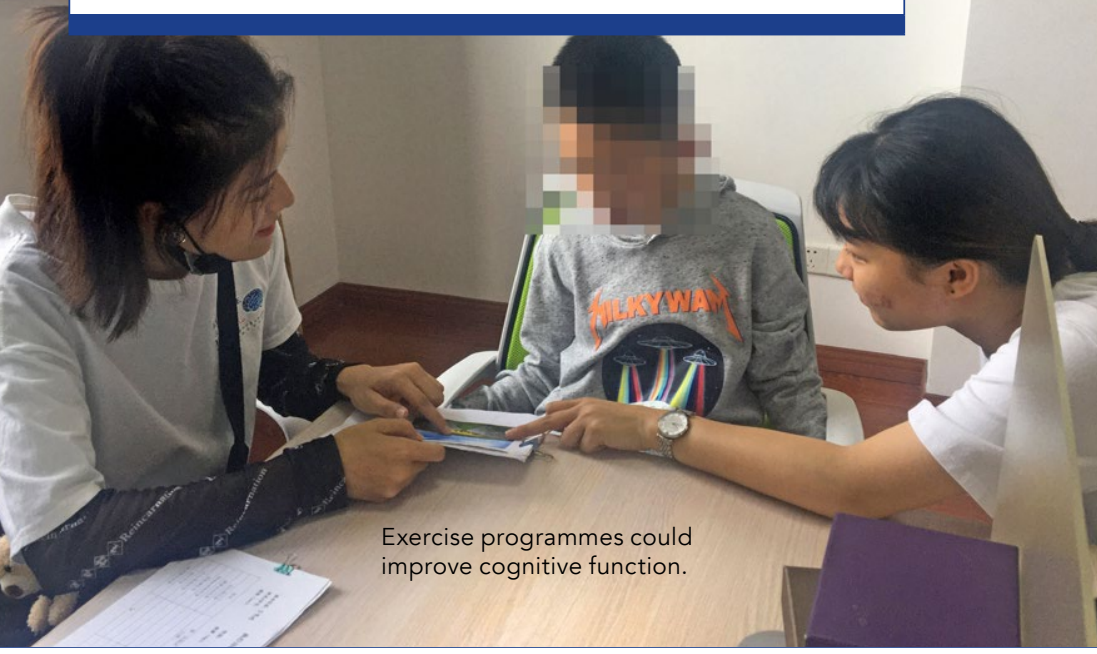

